\title{
Subtyping of Blastocystis sp. isolated from symptomatic and asymptomatic individuals in Makkah, Saudi Arabia
}

\author{
Raafat T. Mohamed ${ }^{1,2^{*}}$, Mohammed A. El-Bali ${ }^{1}$, Anhar A. Mohamed ${ }^{1,3}$, Mona A. Abdel-Fatah ${ }^{3,4}$, \\ Mohamed A. EL-Malky ${ }^{1,5}$, Nawras M. Mowafy ${ }^{1,2}$, Dina A. Zaghlool ${ }^{6,7}$, Rowaida A. Bakri ${ }^{1}$ and Saeed A. Al-Harthi ${ }^{1}$
}

\begin{abstract}
Background: Blastocystis is a group of cosmopolitan gastrointestinal parasite of humans and a wide variety of animals. These anaerobic protozoans include more than 17 specific small-subunit ribosomal RNA subtypes, of which nine are found in humans with a variable geographical distribution. Until now, no study has described the Blastocystis subtypes present in Saudi Arabia.

Methods: In total, 1,262 faecal samples were collected from patients with gastrointestinal complaints and asymptomatic individuals visiting two major hospitals. All samples were analysed by F1/R1 diagnostic PCR, microscopy and culture methods. The subtypes of Blastocystis sp. isolates were determined by the sequenced-tagged site (STS)-based method.

Results: One-hundred-thirty-three positive cases were detected by F1/R1 diagnostic PCR, of which 122 were also positive by the culture method and 83 by direct microscopy. The sensitivities of direct microscopy and the culture method were $62 \%$ and $92 \%$, respectively. Subtype (ST3) was the most prevalent (80.5\%), followed by ST1 (14.5\%) and ST2 (5\%). ST4, ST5, ST6 and ST7 were not detected in this study. ST3 infections were significantly predominant $(P<0.05)$ among symptomatic patients.
\end{abstract}

Conclusions: To our knowledge, this study provides the first run-through information on Blastocystis sp. epidemiology in Makkah city, revealing a rather moderate prevalence of $10.5 \%$ and the presence of three subtypes, ST1, ST2, and ST3. ST3 was the most predominant, particularly among symptomatic patients.

Keywords: Blastocystis sp, Sequence-Tagged Sites (STS) PCR, Subtyping, Makkah city

\section{Background}

Blastocystis is a group of cosmopolitan gastrointestinal anaerobic protozoan parasites. The group is known to infect humans and a wide variety of animal hosts, including mammals, birds, amphibians, and reptiles [1]. The lifecycle comprises numerous 'forms', including granular cysts and multivacuolar, avacuolar, vacuolar, and amoeboid structures. The mode of infection has not been completely understood until now, but involves faecal-oral ingestion of a cyst, with animal handlers showing significantly high rates of infection [2, 3]. Blastocystis has a worldwide

\footnotetext{
* Correspondence: rtmakhlof@uqu.edu.sa

'Department of Medical Parasitology, Faculty of Medicine, Umm Al-Qura University, Makkah, Saudi Arabia

${ }^{2}$ Department of Medical Parasitology, Faculty of Medicine, El-Minia University, El-Minia, Egypt

Full list of author information is available at the end of the article
}

distribution with a marked prevalence in many countries. According to most epidemiological studies, nearly all countries of the world have been classified into welldeveloped, with a moderate prevalence (10-15\%), or under-developed, with a high prevalence (55-70\%), attributed to the levels of hygiene and the presence or absence of contact with animals and/or contaminated water and food $[4,5]$.

Blastocystis may exist in the gut for years; however, the recognition of a high prevalence of Blastocystis in healthy populations using sensitive molecular diagnostic tools has led to a standard shift in clinical Blastocystis research. Studies of the gut microbiota in individuals with and without Blastocystis can provide important information to help determine the role of Blastocystis in human health and disease [6]. Additionally, the Blastocystis parasite appears 
to be more common in healthy individuals than in patients with inflammatory bowel disease and is associated with certain gut microbiota and health indicators. Although the parasite may elicit disease under certain conditions, the focus on Blastocystis may be shifting from a clinical to a public health viewpoint [7]. SSU rRNA-based phylogeny and host origins do not associate with each other for the classification of different Blastocystis isolates that are derived from humans to insects. The Blastocystis sp. subtype (ST) classification is applied for Blastocystis isolates from homoeothermic animals because their origin is from humans, and they are classified into nine genetically different STs. However, the situation in poikilothermic animals, such as reptiles and amphibians, suggests difficulties; therefore, determining the complete sequence of SSU rDNA is essential to establish novel ST in the phylogenetic analysis [8].

In Blastocystis sp. infection, the symptoms are mostly nonspecific and include weight loss, nausea, vomiting, flatulence, abdominal pain, and diarrhoea $[9,10]$. However, the pathogenicity of this parasite is still argumentative and non-conclusive; Blastocystis sp. infections have been reported in several countries in many asymptomatic and apparently healthy individuals as well as in symptomatic cases without any obvious causal organisms [2]. Several studies have deduced that only a few subtypes of Blastocystis sp. can cause symptoms. It was concluded that the proteases of Blastocystis sp. subtype 3 (ST3) were responsible for protein degradation with host immune evasion and are an important virulence factor [11, 12]. Another study correlated Blastocystis sp. subtype 3 infections with idiopathic urticaria [12].

In last few years, several molecular tools have been used to classify the Blastocystis sp. subtypes isolated from humans and animals in different regions worldwide. These techniques include random amplified polymorphic DNA (RAPD) using four different arbitrary polymerase chain reaction (PCR) primers [13] and PCR assays followed by restriction fragment length polymorphism (RFLP) to study the sequence variation in the small-subunit ribosomal RNA genes of isolates [14]. More recently, new PCRbased methods have been developed for subtype classification using known sequenced-tagged site (STS) primer sets [15] in combination with single-strand conformational polymorphism (SSCP) analysis [1] or post-PCR pyrosequencing techniques based on the detection of subtypespecific nucleotide polymorphism in the SSU rRNA gene $[16,17]$, fingerprinting with AP-PCR [18], and multilocus sequence typing [19]. The latest epidemiological studies have shown the great diversity of Blastocystis sp. subtypes; ten subtypes (ST1 to ST10) have been found according to SSU rRNA gene sequences [20]. Seven other subtypes (ST11-ST17) have been identified in isolates from livestock and zoo animals [21]. Among all identified subtypes, only ST1 to ST9 are considered to be human Blastocystis sp. subtypes [10, 22]. Some authors recommend a standardization of Blastocystis terminology to simplify statements and show the relationships of the research results. This primarily depends on the already published data on small-subunit ribosomal RNA gene analyses and suggests that all mammalian and avian isolates should be nominated as Blastocystis sp. [23]. Using Blastocystis genetic markers, the barcode region is the best region to use in publicly available sequence databases at GenBank, while the STS primers represent a suitable approach in situations where sequencing is not an option, and the disadvantages of using STS primers are that some subtypes will go undetected, includes ST8 and ST9 and also the majority of ST4 strains [24].

To our knowledge, no previous study has explored the prevalence and subtype distribution of Blastocystis sp. in Saudi Arabia. The aim of the present study was to explore the Blastocystis sp. relative prevalence and subtypes present in one of the major cities in Saudi Arabia. This survey was performed on faecal samples collected from symptomatic and asymptomatic individuals who visited, over a 10-month period, two major hospitals of Makkah city, Saudi Arabia.

\section{Methods}

\section{Sample collection}

This study was carried out from March 2014 to January 2015 at two major health care centres of Makkah city, King Abdellah medical city and Al-Noor Specialist Hospital. Of all faecal specimens received during this period by Parasitology Laboratory units, in both hospitals, 1,262 specimens were included in this investigation. Approximately $50 \%$ of the collected samples were from patients with gastrointestinal complaints, including severe abdominal pain and diarrhoea, and the other $50 \%$ were from apparently healthy individuals who underwent a regular check-up or mandatory health check.

\section{Microscopic examination and in vitro culture}

The referred faecal specimens are routinely examined by microscopy for parasitic infections using the direct smear method with saline and iodine. Samples from suspected patients, if negative by direct microscopy, are further examined after trichrome permanent staining and according to the formalin ethyl acetate concentration. For this study, each collected sample was split into two parts; one part was kept at $-20{ }^{\circ} \mathrm{C}$, and the other part was inoculated in culture media and transferred to the medical parasitology laboratory of the Faculty of Medicine at Umm Al Qura University for sub-culturing and molecular analysis. The culture medium consisted of Dulbecco's modified Eagle medium (DMEM) (Gibco, Thermo Fisher Scientific, MA, USA) containing $12 \mathrm{mg} /$ 
$\mathrm{ml}$ ampicillin and $4 \mathrm{mg} / \mathrm{ml}$ streptomycin supplemented with $20 \%$ inactivated horse serum (Gibco) sterilized by filtration [25]. The samples were cultured in $11 \times$ $100 \mathrm{~mm}$ sterile screw-capped tubes containing $3 \mathrm{ml}$ of media and were incubated at $37{ }^{\circ} \mathrm{C}$ in an anaerobic gas pack (BD gas pack-Becton, Dickinson, USA). A drop of culture was examined after $72 \mathrm{~h}$ by direct microscopy.

\section{DNA extraction}

Genomic DNA was extracted directly from stool samples using the ZR faecal DNA miniprep kit (Zymo Research, Tustin, CA, USA) and from cell pellets of positive cultures using the QIAmp DNA extraction kit (QIAmp, QIAGEN, Inc., Hilden, Germany), according to manufacturers' protocols. The concentration and purity of isolated DNA were measured by a spectrophotometer (SpectraDrop; SpectroMax, Life Technology, Carlsbad City, CA, USA).

\section{F1/R1 diagnostic PCR}

Diagnostic PCR was applied to genomic DNA extracted from stool specimens using the F1 (5'-GGA GGT AGT GAC AAT AAA TC-3') and R1 (5'-CGT TCA TGA TGA ACA ATT AC-3') primers [26]. Briefly, $2 \mu \mathrm{l}$ of genomic DNA was used in PCR reactions of a $25 \mu \mathrm{l}$ final volume using the AmpliTaq Gold 360 master mix (Applied Biosystems, CA, USA). The PCR conditions consisted of one cycle of initial denaturation at $94{ }^{\circ} \mathrm{C}$ for $4 \mathrm{~min}$, followed by 35 cycles of denaturation at $94{ }^{\circ} \mathrm{C}$ for $30 \mathrm{~s}$, annealing at $54{ }^{\circ} \mathrm{C}$ for $30 \mathrm{~s}$, extension at $72{ }^{\circ} \mathrm{C}$ for $30 \mathrm{~s}$, and a final elongation cycle for $5 \mathrm{~min}$ at $72{ }^{\circ} \mathrm{C}$.

\section{Blastocystis sp. subtyping using Sequence-Tagged Site (STS) primers}

PCR reactions were carried out using sequence-tagged site primer sets derived from the RAPD product sequence to identify the subtypes as follows: ST1 variant (SB82: 5'-TCT TGC TTC ATC GGA GTC / CCT TCT CGC AGT TCT TTA TC-3'), ST1 (SB83: 5'-GAA GGA CTC TCT GAC GAT GA / GTC CAA ATG AAA GGC AGC-3'), ST7 (SB155: 5'-ATC AGC CTA CAA TCT CCT C / ATC GCC ACT TCT CCA AT-3'), ST3 by multiplex PCR including (SB227: 5'-AGG ATT TGG TGT TTG GAG A / TTA GAA GTG AAG GAG ATG GAA G-3', SB228: 5'-GAC TCC AGA AAC TCG CAG AC / TCT TGT TTC CCC AGT TAT CC-3' and SB229: 5'-CAC TGT GTC GTC ATT GTT TTG / AGG GCT GCA TAA TAG AGT GG-3'), ST6 (SB332: 5'-GCA TCC AGA CTA CTA TCA ACA TT / CCA TTT TCA GAC AAC CAC TTA-3'), ST5 (SB336: 5'-GTG GGT AGA GGA AGG AAA ACA / AGA ACA AGT CGA TGA AGT GAG AT-3'), ST4 (SB337: 5'-GTC TTT CCC TGT CTA TTC TTG CA / AAT TCG GTC TGC TTC TTC TG-3'), and ST2 (SB340: 5'-TGT TCT TGT GTC TTC TCA GCT C / TTC TTT CAC ACT CCC GTC AT-3')
[15]. Two microlitres of DNA extract were used in PCR reactions of a $25 \mu \mathrm{l}$ final volume using the AmpliTaq Gold 360 master mix (Applied Biosystems, Carlsbad City, CA, USA). The PCR conditions consisted of one cycle of initial denaturation at $94{ }^{\circ} \mathrm{C}$ for $5 \mathrm{~min}$, followed by 40 cycles that included denaturation at $94{ }^{\circ} \mathrm{C}$ for $30 \mathrm{~s}$, annealing at $57{ }^{\circ} \mathrm{C}$ for $30 \mathrm{~s}$, extension at $72{ }^{\circ} \mathrm{C}$ for $1 \mathrm{~min}$, and a final elongation cycle for $5 \mathrm{~min}$ at $72{ }^{\circ} \mathrm{C}$. All PCR amplifications were carried out in duplicate for each sample and each primer set. The PCR products were separated in 1.5\% agarose gels and were photographed.

\section{Statistical analysis}

The data were analysed using the Chi-square test to compare the frequency of the Blastocystis sp. subtypes from symptomatic and asymptomatic individuals. A $P$-value $<0.05$ was statistically significant. Statistical analysis was performed using SPSS version 21.

\section{Results}

Relative prevalence of Blastocystis sp. based on microscopy and in vitro culture

Of 1,262 examined faecal samples, 133 specimens were found to be positive for Blastocystis by the F1/R1 diagnostic PCR technique used in this study. Among positive cases, 85 (64\%) were patients with gastrointestinal symptoms and $48(36 \%)$ were asymptomatic individuals. The general symptoms consisted of abdominal pain in approximately $76 \%$, diarrhoea in approximately $27 \%$ and other gastrointestinal complaints in approximately $16 \%$ of the symptomatic patients. All 133 positive cases were detected by F1/R1 diagnostic PCR, of which 122 were also positive by the culture method and 83 by direct microscopy. The sensitivities of direct microscopy and the culture methods compared with that of F1/R1 PCR and are shown in Table 1.

\section{STS subtyping analysis of Blastocystis sp. isolates}

Three Blastocystis sp. subtypes (ST1, ST2 and ST3) were detected by STS primer-based PCR analysis of positive samples. ST3 was the most prevalent (80.5\%), followed by ST1 (14.5\%) and ST2 (5\%). ST4, ST5, ST6 and ST7 were not detected in this study. ST3 infections were significantly predominant $\left(\chi^{2}=6.54, \quad d f=2, \quad P=0.038\right)$ among symptomatic patients (Fig. 1). The Blastocystis sp. subtype distribution did not show a predisposition in relation to the age or gender of infected individuals.

\section{Discussion}

In this study, the relative prevalence of Blastocystis sp. infection among individuals with or without gastrointestinal symptoms who visited two major hospitals in Makkah city was determined to be $10.5 \%$. Since the prevalence data are usually derived from general surveys 
Table 1 Sensitivity of F1/R1 PCR, direct microscopy, and the culture method for the diagnosis of Blastocystis sp. infections in the stool samples of patients with Gl symptoms and asymptomatic individuals

\begin{tabular}{lllll}
\hline $\begin{array}{l}\text { Diagnostic } \\
\text { technique }\end{array}$ & $\begin{array}{l}\text { Symptomatic } \\
(n=85)\end{array}$ & $\begin{array}{l}\text { Asymptomatic } \\
(n=48)\end{array}$ & $\begin{array}{l}\text { Total } \\
(n=133)\end{array}$ & Sensitivity \\
\hline Direct microscopy & 65 & 18 & 83 & $62 \%$ \\
In vitro culture & 79 & 43 & 122 & $92 \%$ \\
F1/R1 PCR & 85 & 48 & 133 & $100 \%$ \\
\hline
\end{tabular}

of intestinal parasites using the formol acetate concentration technique with microscopic examination, such numbers should be interpreted with extreme caution because the sensitivities of this method are lower than those of nucleic acid-based methods, including conventional and real-time PCR [7]. Epidemiological studies in several countries with different sanitation standards revealed wide ranges of Blastocystis sp. prevalence: $0.54 \%$ in Turkey [27], 2.5\% in Japan [15], 3.3\% in Singapore [28], 19\% in Lebanon [29], 22.1\% in Libya [30], 33.3\% in Egypt [31], 40.7\% in the Philippines [32] and $46.9 \%$ in Venezuela [33]. Thus, the prevalence found herein is relatively moderate compared with the worldwide general infection situation.

Numerous conflicting reports on the pathogenic capability of Blastocystis sp. infection have been published, and it is still under debate whether Blastocystis sp. is a pathogenic or a commensal life form [2]. In our study, Blastocystis infections were detected both in patients with patent gastrointestinal symptoms ( 85 cases) and in apparently healthy individuals (48) who underwent mandatory health check-ups. Additionally, $78.5 \%$ of symptomatic patients and $21.5 \%$ of asymptomatic individuals with PCR-positive infections were positive by microscopy; accordingly, it has been reported that the Blastocystis cell density is usually significantly higher in symptomatic patients [34]. Comparative studies of human immunity against Blastocystis infection have revealed a significant difference in antibody responses between symptomatic and asymptomatic individuals $[35,36]$. However, antigenic heterogeneity has been detected between Blastocystis sp. isolated from symptomatic patients and asymptomatic individuals in axenic culture; however, these studies did not explore any possible correlations between this heterogeneity and genetic subtypes [37].

Nine subtypes of Blastocystis sp. (ST1 to ST9) were identified in humans [10]. To our knowledge, this is the first report exploring the subtypes of Blastocystis in Makkah city, Saudi Arabia. Using sequence-tagged site (STS) primer-based PCR, we found three Blastocystis sp. subtypes (ST1, ST2 and ST3) among the 133 positive cases. The high predominance of ST3 $(80.5 \%)$ in our study population agrees with different reports in the literature; ST3 was found to be the most dominant subtype, varying from $41 \%$ to $92 \%$, in a comparative study between Japan, Bangladesh, Pakistan and Germany [15] and at 78\%, $75.9 \%, 54.5 \%, 53.5 \%$ and $33.3 \%$ in Singapore, Turkey, Egypt, France and Lebanon, respectively [28, 29, 38-40].

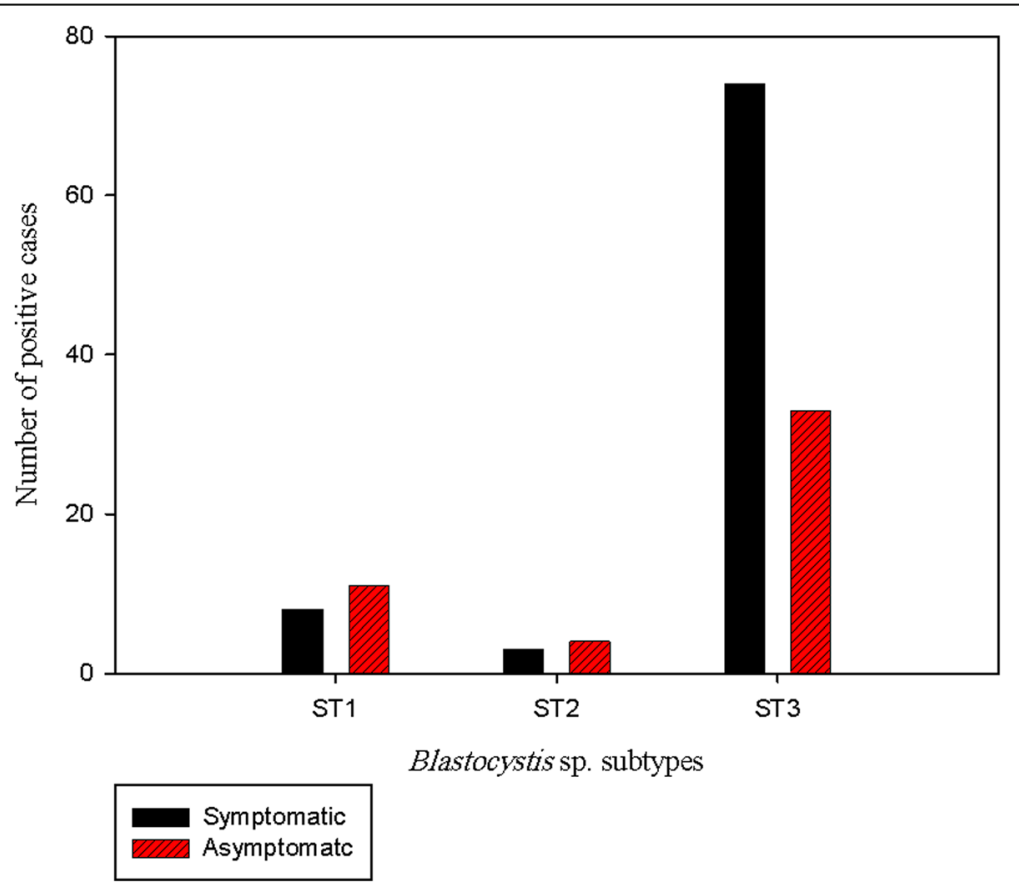

Fig. 1 Subtype distribution of Blastocystis sp. among gastrointestinal patients and asymptomatic individuals. Subtype 3 was $69 \%$ in symptomatic patients and $31 \%$ in asymptomatic individuals $\left(X^{2}=6.54, d f=2, P<0.05\right)$ 
However, the ST1 (50\%) subtype was found to be more predominant than ST3 (39.5\%) in the Libyan population [41], as confirmed by a more recent study reporting the predominance of ST1 (51.1\%) over ST3 (17.8\%) in Libya [30]. Although ST2 is commonly reported to be the second most prevalent subtype [42-45], in our study, only $5 \%$ of cases carried the ST2 subtype, while 14.5\% carried ST1.

Subtypes ST4, ST5, ST6 and ST7, as reported in previous studies [39, 46, 47], were not detected in our investigation. ST4 was remarkably common in Danish patients with acute diarrhoea [48], symptomatic patients in Spain [43], and rural communities in Nepal [49]. ST6 and ST7 were reported in the Middle East both in irritable bowel syndrome patients and healthy persons [39, 47]. However, ST5 was mostly reported in animals and humans living near to farms $[16,50]$.

In many previous studies, no association was reported between the specific subtype of Blastocystis sp. and explicit gastrointestinal symptoms [40, 46, 51]. However, other studies reported a specific association between subtypes of the parasite and apparent symptoms: it was demonstrated that Blastocystis cysteine proteinase can degrade human IgA [52]. Additionally, patients with chronic gastrointestinal illness with reported antibiotic failure for over four years were diagnosed with ST3 and ST1 infections [53]. More recently, irritable bowel syndrome in several Mexican patients was associated with Blastocystis sp. ST1 and ST3 infections [54]. Consistently, ST3 infections were significantly predominant among symptomatic patients (69\%) in our study. Blastocystis sp. ST2 infection was linked to gastrointestinal illness and chronic urticaria [55].

In our findings, this subtype did not show an apparent predominance in distribution between symptomatic and asymptomatic infected individuals. However, it appears that the Blastocystis sp. infection clinical outcome is multifactorial, complicating the evaluation of its pathogenicity, even during case-controlled studies [5].

\section{Conclusions}

The outcome of this study provides the first runthrough information on Blastocystis sp. epidemiology in Makkah city, revealing a relatively moderate prevalence $(10.5 \%)$, as well as the presence of three subtypes: ST3, the most predominant particularly among symptomatic patients; ST1; and ST2. Further screenings are needed to clarify the epidemiology of this gastrointestinal parasite in local and emigrant populations of the entire country. Likewise, the present study underlines the advantage of STS-PCR as a significant technique for Blastocystis sp. subtyping in epidemiological studies.

\section{Abbreviations}

AP-PCR: Arbitrary primer polymerase chain reaction; DMEM: Dulbecco's modified Eagle medium; RAPD: Random amplified polymorphic DNA; RFLP: Restriction fragment length polymorphism; SSCP: Single strand conformational polymorphisms; SSU rRNA: Small-subunit of the ribosomal RNA; STS: Sequence-tagged site; STs: Subtypes

\section{Acknowledgements \\ We would like to thank the Faculty of Medicine at Umm Al Qura University for providing research facilities for this work. This publication was made possible by the support of the ISR program of UQU, Makkah, Saudi Arabia (Grant No. 43409049).}

\section{Funding}

This research was funded by the ISR program of Umm Al-Qura University, Makkah (Grant No. 43409049). The funding body played no role in designing the research study or collection, analysis and interpretation or even in writing the manuscript.

\section{Availability of data and materials}

The dataset supporting the conclusions of this article is included within the article.

\section{Authors' contributions}

RTM, MAE and AAM conceived the study, carried out in vitro culture, performed subtyping PCR, and wrote the manuscript. MAA and DAZ collected the patient data and stool samples. MAA, MAE, NMM and DAZ processed the stool specimens and performed microscopic examination. SAA and RAB analysed the data and participated in the writing of the manuscript. All authors read and approved the final manuscript.

\section{Competing interests}

The authors declare that they have no competing interests.

\section{Consent for publication}

Not applicable.

\section{Ethics approval and consent to participate}

Ethical approval for this project was obtained from the Medical Research Centre and Research Committee at the Faculty of Medicine, Umm Al-Qura University, Saudi Arabia (Research protocol\# 43409049). All participants who joined this research had signed an informed consent.

\section{Publisher's Note}

Springer Nature remains neutral with regard to jurisdictional claims in published maps and institutional affiliations.

\section{Author details}

${ }^{1}$ Department of Medical Parasitology, Faculty of Medicine, Umm Al-Qura University, Makkah, Saudi Arabia. ${ }^{2}$ Department of Medical Parasitology, Faculty of Medicine, El-Minia University, El-Minia, Egypt. ${ }^{3}$ Department of Medical Parasitology, Faculty of Medicine, Ain Shams University, Cairo, Egypt. ${ }^{4}$ Microbiology Laboratory, King Abdellah Medical City, Makkah, Saudi Arabia. ${ }^{5}$ Department of Medical Parasitology, Faculty of Medicine, Mansoura University, Mansoura, Egypt. 'Laboratory and Blood Bank Department, Al-Noor Specialist Hospital, Makkah, Saudi Arabia. ${ }^{7}$ Department of Medical Parasitology, Faculty of Medicine, Assiut University, Assiut, Egypt.

Received: 28 July 2016 Accepted: 27 March 2017

Published online: 07 April 2017

References

1. Menounos PG, Spanakos G, Tegos N, Vassalos CM, Papadopoulou C, Vakalis NC. Direct detection of Blastocystis sp. in human faecal samples and subtype assignment using single strand conformational polymorphism and sequencing. Mol Cell Probes. 2008;22(1):24-9.

2. Stenzel DJ, Boreham PF. Blastocystis hominis revisited. Clin Microbiol Rev. 1996;9(4):563-84.

3. Yoshikawa H, Abe N, Iwasawa M, Kitano S, Nagano I, Wu Z, Takahashi Y. Genomic analysis of Blastocystis hominis strains isolated from two long-term health care facilities. J Clin Microbiol. 2000;38(4):1324-30. 
4. Forsell J, Granlund M, Stensvold CR, Clark CG, Evengard B. Subtype analysis of Blastocystis isolates in Swedish patients. Eur J Clin Microbiol Infect Dis. 2012;31(7):1689-96.

5. Tan KS. New insights on classification, identification, and clinical relevance of Blastocystis spp. Clin Microbiol Rev. 2008;21(4):639-65.

6. Stensvold CR, Clark CG. Current status of Blastocystis: A personal view. Parasitol Int. 2016;65(6 Pt B):763-71.

7. Andersen LO, Stensvold CR. Blastocystis in Health and Disease. Are we moving from a clinical to a public health perspective? J Clin Microbiol. 2016;54(3):524-8.

8. Yoshikawa H, Koyama Y, Tsuchiya E, Takami K. Blastocystis phylogeny among various isolates from humans to insects. Parasitol Int. 2016;65(6 Pt B):750-9.

9. Tasova Y, Sahin B, Koltas S, Paydas S. Clinical significance and frequency of Blastocystis hominis in Turkish patients with hematological malignancy. Acta Med Okayama. 2000;54(3):133-6.

10. Rene BA, Stensvold CR, Badsberg JH, Nielsen HV. Subtype analysis of Blastocystis isolates from Blastocystis cyst excreting patients. Am J Trop Med Hyg. 2009; 80(4):588-92.

11. Abdel-Hameed DM, Hassanin OM. Proteaese activity of Blastocystis hominis subtype3 in symptomatic and asymptomatic patients. Parasitol Res. 2011; 109(2):321-7.

12. Hameed DM, Hassanin OM, Zuel-Fakkar NM. Association of Blastocystis hominis genetic subtypes with urticaria. Parasitol Res. 2011;108(3):553-60.

13. Yoshikawa H, Nagano I, Wu Z, Yap EH, Singh M, Takahashi Y. Genomic polymorphism among Blastocystis hominis strains and development of subtype-specific diagnostic primers. Mol Cell Probes. 1998;12(3):153-9.

14. Clark CG. Extensive genetic diversity in Blastocystis hominis. Mol Biochem Parasitol. 1997;87(1):79-83.

15. Yoshikawa H, Wu Z, Kimata I, Iseki M, Ali IK, Hossain MB, et al. Polymerase chain reaction-based genotype classification among human Blastocystis hominis populations isolated from different countries. Parasitol Res. 2004; 92(1):22-9.

16. Yan Y, Su S, Ye J, Lai X, Lai R, Liao H, et al. Blastocystis sp. subtype 5: a possibly zoonotic genotype. Parasitol Res. 2007;101(6):1527-32.

17. Stensvold CR, Traub RJ, von Samson-Himmelstjerna G, Jespersgaard C, Nielsen HV, Thompson RC. Blastocystis: subtyping isolates using pyrosequencing technology. Exp Parasitol. 2007;116(2):111-9.

18. Tan TC, Suresh KG, Thong KL, Smith HV. PCR fingerprinting of Blastocystis isolated from symptomatic and asymptomatic human hosts. Parasitol Res. 2006;99(4):459-65.

19. Arisue N, Hashimoto T, Yoshikawa H, Nakamura Y, Nakamura G, Nakamura F, et al. Phylogenetic position of Blastocystis hominis and of stramenopiles inferred from multiple molecular sequence data. J Eukaryot Microbiol. 2002; 49(1):42-53.

20. Stensvold CR, Alfellani MA, Norskov-Lauritsen S, Prip K, Victory EL, Maddox C, et al. Subtype distribution of Blastocystis isolates from synanthropic and zoo animals and identification of a new subtype. Int J Parasitol. 2009;39(4):473-9.

21. Alfellani MA, Taner-Mulla D, Jacob AS, Imeede CA, Yoshikawa H, Stensvold CR, Clark CG. Genetic diversity of blastocystis in livestock and zoo animals. Protist. 2013:164(4):497-509.

22. Parkar U, Traub RJ, Vitali S, Elliot A, Levecke B, Robertson I, et al. Molecular characterization of Blastocystis isolates from zoo animals and their animalkeepers. Vet Parasitol. 2010;169(1-2):8-17.

23. Stensvold CR, Suresh GK, Tan KS, Thompson RC, Traub RJ, Viscogliosi E, et al. Terminology for Blastocystis subtypes-a consensus. Trends Parasitol. 2007; 23(3):93-6.

24. Stensvold CR. Comparison of sequencing (barcode region) and sequencetagged-site PCR for Blastocystis subtyping. J Clin Microbiol. 2013:51(1):190-4.

25. Zhang X, Qiao JY, Da R, Li YQ, Yao FR. In vitro culture of Blastocystis hominis in medium DMEM. Wei Sheng Yan Jiu. 2006;35(6):743-6 (In Chinese).

26. Bohm-Gloning B, Knobloch J, Walderich B. Five subgroups of Blastocystis hominis from symptomatic and asymptomatic patients revealed by restriction site analysis of PCR-amplified 16S-like rDNA. Trop Med Int Health. 1997;2(8):771-8

27. Beyhan YE, Yilmaz H, Cengiz ZT, Ekici A. Clinical significance and prevalence of Blastocystis hominis in Van, Turkey. Saudi Med J. 2015;36(9):1118-21.

28. Wong KH, Ng GC, Lin RT, Yoshikawa H, Taylor MB, Tan KS. Predominance of subtype 3 among Blastocystis isolates from a major hospital in Singapore. Parasitol Res. 2008;102(4):663-70.

29. El Safadi D, Meloni D, Poirier P, Osman M, Cian A, Gaayeb L, et al. Molecular epidemiology of Blastocystis in Lebanon and correlation between subtype 1 and gastrointestinal symptoms. Am J Trop Med Hyg. 2013;88(6):1203-6.
30. Abdulsalam AM, Ithoi I, Al-Mekhlafi HM, Al-Mekhlafi AM, Ahmed A, Surin J. Subtype distribution of Blastocystis isolates in Sebha. Libya PloS one. 2013; 8(12):e84372.

31. Rayan HZ, Ismail OA, El Gayar EK. Prevalence and clinical features of Dientamoeba fragilis infections in patients suspected to have intestinal parasitic infection. J Egypt Soc Parasitol. 2007;37(2):599-608.

32. Baldo ET, Belizario W, De Leon WU, Kong HH, Chung DI. Infection status of intestinal parasites in children living in residential institutions in Metro Manila, the Philippines. Korean J Parasitol. 2004;42(2):67-70.

33. Velasquez V, Caldera R, Wong W, Cermeno G, Fuentes M, Blanco Y, et al. Blastocystosis: a high prevalence of cases found in patients from Health Center of Soledad, Anzoategui State, Venezuela. Rev Soc Bras Med Trop. 2005:38(4):356-7 (In Spanish).

34. Al-Fellani MA, Khan AH, Al-Gazoui RM, Zaid MK, Al-Ferjani MA. Prevalence and clinical features of Blastocystis hominis infection among patients in Sebha, Libya. Sultan Qaboos Univ Med J. 2007;7(1):35-40.

35. Zierdt CH, Zierdt WS, Nagy B. Enzyme-linked immunosorbent assay for detection of serum antibody to Blastocystis hominis in symptomatic infections. J Parasitol. 1995:81(1):127-9.

36. Hussain R, Jaferi W, Zuberi S, Baqai R, Abrar N, Ahmed A, Zaman V. Significantly increased lgG2 subclass antibody levels to Blastocystis hominis in patients with irritable bowel syndrome. Am J Trop Med Hyg. 1997;56(3):301-6.

37. Lanuza MD, Carbajal JA, Villar J, Mir A, Borras R. Soluble-protein and antigenic heterogeneity in axenic Blastocystis hominis isolates: pathogenic implications. Parasitol Res. 1999;85(2):93-7.

38. Ozyurt M, Kurt O, Molbak K, Nielsen HV, Haznedaroglu T, Stensvold CR. Molecular epidemiology of Blastocystis infections in Turkey. Parasitol Int 2008:57(3):300-6.

39. Hussein EM, Hussein AM, Eida MM, Atwa MM. Pathophysiological variability of different genotypes of human Blastocystis hominis Egyptian isolates in experimentally infected rats. Parasitol Res. 2008;102(5):853-60.

40. Souppart L, Sanciu G, Cian A, Wawrzyniak I, Delbac F, Capron M, et al. Molecular epidemiology of human Blastocystis isolates in France. Parasitol Res. 2009;105(2):413-21.

41. Alfellani MA, Stensvold CR, Vidal-Lapiedra A, Onuoha ES, Fagbenro-Beyioku AF, Clark CG. Variable geographic distribution of Blastocystis subtypes and its potential implications. Acta Trop. 2013;126(1):11-8.

42. Dogruman-Al F, Dagci H, Yoshikawa H, Kurt O, Demirel M. A possible link between subtype 2 and asymptomatic infections of Blastocystis hominis. Parasitol Res. 2008;103(3):685-9.

43. Dominguez-Marquez MV, Guna R, Munoz C, Gomez-Munoz MT, Borras R. High prevalence of subtype 4 among isolates of Blastocystis hominis from symptomatic patients of a health district of Valencia (Spain). Parasitol Res. 2009;105(4):949-55.

44. Yoshikawa H, Wu Z, Pandey K, Pandey BD, Sherchand JB, Yanagi T, Kanbara H. Molecular characterization of Blastocystis isolates from children and rhesus monkeys in Kathmandu, Nepal. Vet Parasitol. 2009:160(3-4):295-300.

45. Meloni D, Sanciu G, Poirier P, El Alaoui H, Chabe M, Delhaes L, et al. Molecular subtyping of Blastocystis sp. isolates from symptomatic patients in Italy. Parasitol Res. 2011;109(3):613-9.

46. Yakoob J, Jafri W, Jafri N, Khan R, Islam M, Beg MA, Zaman V. Irritable bowe syndrome: in search of an etiology: role of Blastocystis hominis. Am J Trop Med Hyg. 2004;70(4):383-5.

47. Yakoob J, Jafri W, Beg MA, Abbas Z, Naz S, Islam M, Khan R. Irritable bowel syndrome: is it associated with genotypes of Blastocystis hominis. Parasitol Res. 2010;106(5):1033-8.

48. Stensvold CR, Christiansen DB, Olsen KE, Nielsen HV. Blastocystis sp. subtype 4 is common in Danish Blastocystis-positive patients presenting with acute diarrhea. Am J Trop Med Hyg. 2011;84(6):883-5.

49. Lee IL, Tan TC, Tan PC, Nanthiney DR, Biraj MK, Surendra KM, Suresh KG Predominance of Blastocystis sp. subtype 4 in rural communities, Nepal. Parasitol Res. 2012:110(4):1553-62.

50. Petrasova J, Uzlikova M, Kostka M, Petrzelkova KJ, Huffman MA, Modry D. Diversity and host specificity of Blastocystis in syntopic primates on Rubondo Island, Tanzania. Int J Parasitol. 2011;41(11):1113-20.

51. Motazedian H, Ghasemi H, Sadjjadi SM. Genomic diversity of Blastocystis hominis from patients in southern Iran. Ann Trop Med Parasitol. 2008;102(1):85-8.

52. Puthia MK, Vaithilingam A, Lu J, Tan KS. Degradation of human secretory immunoglobulin A by Blastocystis. Parasitol Res. 2005;97(5):386-9.

53. Jones MS, Whipps CM, Ganac RD, Hudson NR, Boorom K. Association of Blastocystis subtype 3 and 1 with patients from an Oregon community 
presenting with chronic gastrointestinal illness. Parasitol Res. 2009;104(2): 341-5.

54. Jimenez-Gonzalez DE, Martinez-Flores WA, Reyes-Gordillo J, Ramirez-Miranda ME, Arroyo-Escalante S, Romero-Valdovinos M, et al. Blastocystis infection is associated with irritable bowel syndrome in a Mexican patient population. Parasitol Res. 2012;110(3):1269-75.

55. Vogelberg C, Stensvold CR, Monecke S, Ditzen A, Stopsack K, Heinrich-Grafe U, Pohlmann C. Blastocystis sp. subtype 2 detection during recurrence of gastrointestinal and urticarial symptoms. Parasitol Int. 2010;59(3):469-71.

Submit your next manuscript to BioMed Central and we will help you at every step:

- We accept pre-submission inquiries

- Our selector tool helps you to find the most relevant journal

- We provide round the clock customer support

- Convenient online submission

- Thorough peer review

- Inclusion in PubMed and all major indexing services

- Maximum visibility for your research

Submit your manuscript at www.biomedcentral.com/submit 